\title{
MODELAGEM DA HIDRÓLISE ENZIMÁTICA NO BAGAÇO DE CANA-DE-AÇÚCAR PARA PRODUÇÃO DE GLICOSE
}

\author{
A. DE A. GUILHERME ${ }^{1}$, P. V. F. DANTAS ${ }^{1}$, E. S. DOS SANTOS ${ }^{1}$, F. A. N. FERNANDES ${ }^{2}$, \\ G. R. DE MACEDO ${ }^{1}$ \\ ${ }^{1}$ Universidade Federal do Rio Grande do Norte - Departamento de Engenharia Química \\ ${ }^{2}$ Universidade Federal do Ceará - Departamento de Engenharia Química \\ Contato: alexandredearaujoguilherme@gmail.com
}

\begin{abstract}
RESUMO:este trabalho teve como objetivo a modelagem do processo de hidrólise enzimática utilizando bagaço de cana-de-açúcar como substrato para produção de glicose. Foram realizados ensaios cinéticos variando concentração inicial de substrato e atividades enzimáticas, ensaios para a obtenção da constante de adsorção de acordo com o modelo de Langmuir, ensaios para avaliação da reatividade do substrato, para obtenção de energia de ativação da enzima e ensaios de inibições causadas pela glicose, celobiose e xilosena hidrólise enzimática. $\mathrm{O}$ sistema de equações diferenciais foi integrado numericamente utilizando o método matemático de integração numérica Runge-Kutta de $5^{\mathrm{a}}$ ordem. A estimativa dos parâmetros foi realizada segundo o método de Levenberg-Marquardt de regressão nãolinear.De acordo com os resultados, foi possível o ajuste do modelo aos dadosexperimentais. Com este modelo é possível se fazer uma otimização do processo de hidrólise enzimática bem como utiliza-lo como parte da modelagem do processo de sacarificação e fermentação simultânea para produção de etanol.
\end{abstract}

\section{INTRODUÇÃO}

Para a produção de etanol de segunda geração (etanol celulósico) é preciso que se obtenham monômeros de carboidratos como a glicose proveniente da estrutura da parede celular vegetal, lignocelulose. Desta forma, diferentemente do processo convencional, para a produção de etanol de segunda geração é preciso que o material lignocelulósico seja submetido a um pré-tratamento químico, físico, biológico ou combinado e a uma hidrólise enzimática para que se tenha substrato disponível para a fermentação alcoólica.

Na hidrólise enzimática da celulose são necessário pelo menos três tipos de enzimas para se que se obtenha glicose. As endoglicanases (EG) (EC.3.2.1.4) hidrolisam as moléculas de celulose de forma randômica produzindo oligômeros. As celobiohidrolases ou exoglicanases $(\mathrm{CBH})$ (EC.3.2.1.91) convertem os oligômeros, formados pela ação das endoglicanases, em celobiose e celodextrinas solúveis. A $\beta$-glicosidase (BG), também chamada de celobiase, (EC.3.2.1.21) converte celobiose e celodextrinas em glicose (Zhouet al., 2009). As enzimas celobiohidrolases agem nas extremidades dos oligômeros sendo então de dois tipos, uma que age na extremidade redutora e o outro tipo que age na extremidade não-redutora (Lyndet al., 2002). Todas estas enzimas trabalham sinergicamente para hidrolisar a celulose (GalbeeZacchi, 2002). 
Vários são os trabalhos realizados em diferentes biomassas vegetais para a modelagem da hidrólise enzimática visando à produção de glicose (BelkacemiandHamoudi, 2003;Kadamet al., 2004; Liaoet al., 2008; Tinget al., 2009; Morales-Rodriguezet al., 2011;Khodaverdiet al., 2012).

Este trabalho teve como objetivo a modelagem matemática do processo de hidrólise enzimática utilizando o bagaço de cana de açúcar como substrato para a produção de glicose utilizando um modelo fenomenológico baseado em dados experimentais.

\section{MATERIAL E MÉTODOS}

\subsection{Enzimas e bagaço de cana-de-açúcar}

A enzimautilizada neste trabalho foi cedida gentilmente pela Novozymes, e foi o complexo de celulases NS22086 (220 FPU/mL, 4500 CBU/mL(Ghose, 1987) e 0,04 g/mL de proteína total).O substrato utilizado neste trabalho foi o bagaço de cana-de-açúcar cedido gentilmente pela Usina Estivas (Arês - RN, Brasil) e submetido a um pré-tratamento com ácido sulfúrico diluído em uma primeira etapa (Guoet al., 2009)e hidróxido de sódio em uma segunda etapa(Vasquez et al., 2007) e analisado segundo (Gouveia et al., 2009) para determinação da composição química.

\subsection{Ensaios enzimáticos}

Foram realizados ensaios cinéticos variando a atividade enzimática inicial de 5 a 90 FPU/g celulose e a quantidade de celulose inicial de 2 a $6 \%(\mathrm{~m} / \mathrm{m})$. O complexo enzimático utilizado já possui atividade celobiase suficiente, portanto, não precisou ser suplementada com enzimas $\beta$-glicosidase. Os ensaiosforam realizados em incubador rotatório com $150 \mathrm{rpm}$ e $50^{\circ} \mathrm{C}$ por 12 horas utilizando Erlenmyers de $250 \mathrm{~mL}$ e $100 \mathrm{~mL}$ de volume reacional com tampão citrato $50 \mathrm{mM}$ em pH 4,8. Uma solução de azida de sódio $1 \%(\mathrm{~m} / \mathrm{m})$ na proporção de $1: 100(\mathrm{v} / \mathrm{m})$ foi usada para prevenir o crescimento de micro-organismos.

Para os ensaios de adsorção(Khodaverdiet al., 2012) foi usada à quantidade de celulose de $1 \%$ em erlenmyer de $250 \mathrm{mLcom} 20 \mathrm{~mL}$ de volume reacional, e as mesmas atividades enzimáticas utilizadas nos ensaios cinéticos. Para os ensaios de reatividade (Khodaverdiet al., 2012), energia de ativação (Kadamet al., 2004) e inibição, foi utilizada a quantidade de $3 \%$ de celulose em erlenmyer de $250 \mathrm{mLcom} 20 \mathrm{~mL}$ de volume reacional e $15 \mathrm{FPU} / \mathrm{g}$ celulose das enzimas celulases.Nos ensaios de inibiçãoforam usadas celulose do bagaço de cana ou celobiose pura como substrato, as inibições testadas foram de acordo com a literatura (Kadamet al., 2004; MoralesRodriguezet al., 2011;Khodaverdiet al., 2012) e estão apresentados na Tabela 1.

Tabela 1. Condições de inibidores, substrato e tempo para os ensaios de inibição.

\begin{tabular}{cccc}
\hline Condições & $\begin{array}{c}\text { Reação: celulose }- \\
\text { celobiose }\end{array}$ & $\begin{array}{c}\text { Reação: celulose - } \\
\text { glicose }\end{array}$ & $\begin{array}{c}\text { Reação: celobiose - } \\
\text { glicose }\end{array}$ \\
\hline Celobiose $(\mathrm{g} / \mathrm{L})$ & $0,01,0,15$ e 0,3 & 100,132 e 140 & $20,24,3$ e 30 \\
Glicose $(\mathrm{g} / \mathrm{L})$ & $0,05,0,1$ e 0,2 & $0,02,0,04$ e 0,06 & $1,5,3,9$ e 5 \\
Xilose $(\mathrm{g} / \mathrm{L})$ & $0,05,0,1$ e 0,2 & $0,05,0,2$ e 0,35 & 180,201 e 220 \\
Substrato & Celulose & Celulose & Celobiose \\
Tempo & 30 min. & $3 \mathrm{~h}$ & $2 \mathrm{~h}$ \\
\hline
\end{tabular}




\subsection{Modelagem matemática}

O modelo proposto foi baseado nos modelos publicados na literatura com algumas modificações tomando-se por base os resultados dos ensaios de inibição e alguns fenômenos ocorridos na hidrólise enzimática. O software utilizado para a modelagem foi o Intel Fortran v. 11.0. O sistema de equações diferenciais foi integrado numericamente utilizando o método matemático de integração numérica Runge-Kutta de $5^{\mathrm{a}}$ ordem. A estimativa dos parâmetros foi realizada segundo o método de Levenberg-Marquardt de regressão não-linear para os parâmetros não estimados experimentalmente.O modelo foi validado estatisticamente através do teste do $X^{2}(\alpha=0,5)$ realizado no software STATISTICA 7.0 para se comparar os dados experimentais contra os preditos pelo modelo.

\section{RESULTADOS E DISCUSSÃO}

Para a composição do bagaço de cana foi encontrado $65 \%$ de celulose, $9,6 \%$ de hemicelulose e 7,8\% de lignina. Na Figura 1 estão apresentados os resultados para os ensaios cinéticos para a produção de glicose e xilose nas diferentes condições de atividade enzimática inicial, celulose inicial e um ensaio realizado com bagaço de cana sem pré-tratamento.
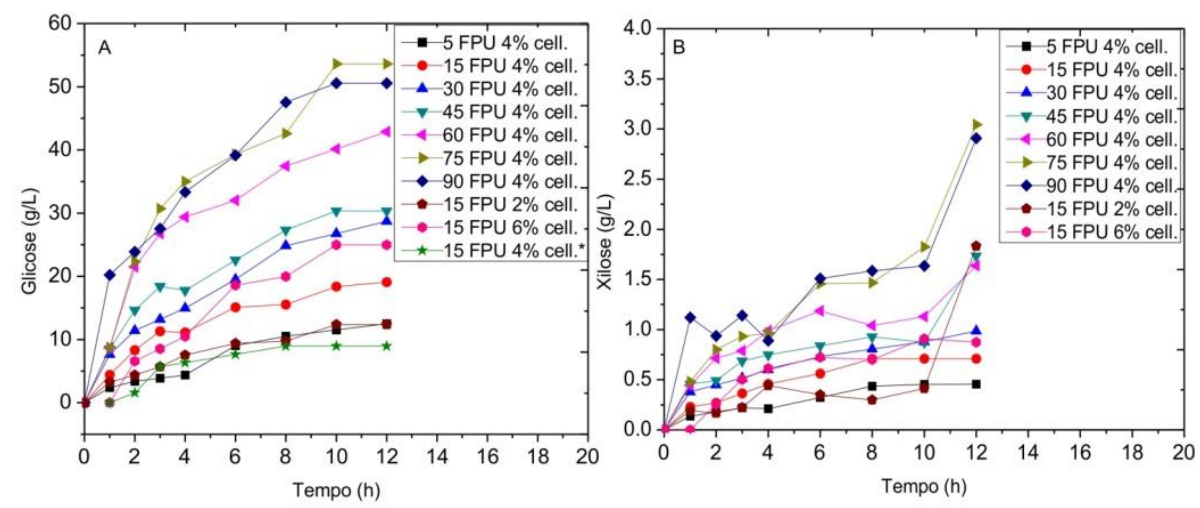

*Hidrólise com bagaço de cana sem pré-tratamento.

Figura 1. A - produção de glicose, B - produção de xilose, em diferentes condições de hidrólise.

Pode-se observar que a produção de glicose aumenta na medida em que se aumenta a quantidade de substrato e atividade enzimática inicial até o limite de 75 FPU, depois a produção se mantem constante e que a produção de glicose a partir de bagaço não pré-tratado é inferior em comparação com o bagaço pré-tratado. Na produção de xilose observa-se um acréscimo repentino na produção a partir de 10 hs de processo para os ensaios com maiores razões (enzima/substrato). Este é um resultado inusitado para se pensar como o complexo enzimático age na produção de xilose, se existe uma pequena atividade enzimática no mix enzimático ou se essa xilose é liberada no meio de forma indireta devido à ação das outras enzimas desestruturando a matriz lignocelulósica. Entretanto, observando a concentração de hemicelulose no bagaço pré-tratado que foi de $29 \mathrm{~g} / \mathrm{L}$ e as quantidades baixas de xilose produzida pode-se imaginar que o que ocorre é uma liberação dessa xilose no meio devido à ação indireta das enzimas atuando na matriz lignocelulósica. Os resultados cinéticos para 
celulose, celobiose e hemicelulose não estão demonstrados neste trabalho. Na Figura 2 estão apresentados os resultados para o ensaio de adsorção e reatividade do substrato e na Figura 3 estão apresentados os resultados de inibição para a reação (celulose-glicose) e os resultados do ajuste da modelagem para a condição de $15 \mathrm{FPU} / \mathrm{g}$ celulose e $4 \%$ de celulose inicial.
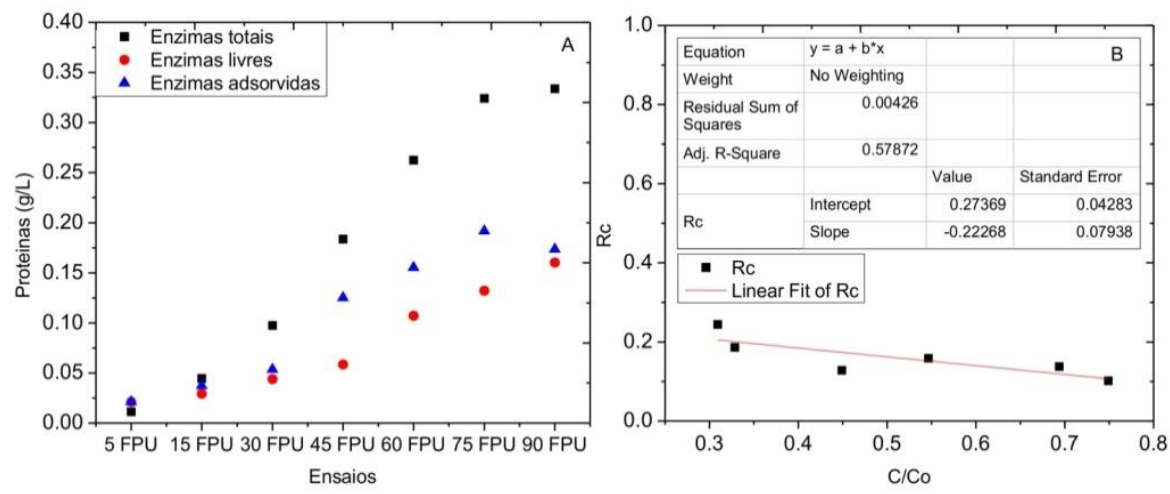

Figura 2. A - ensaios de adsorção, B - ensaios de reatividade.
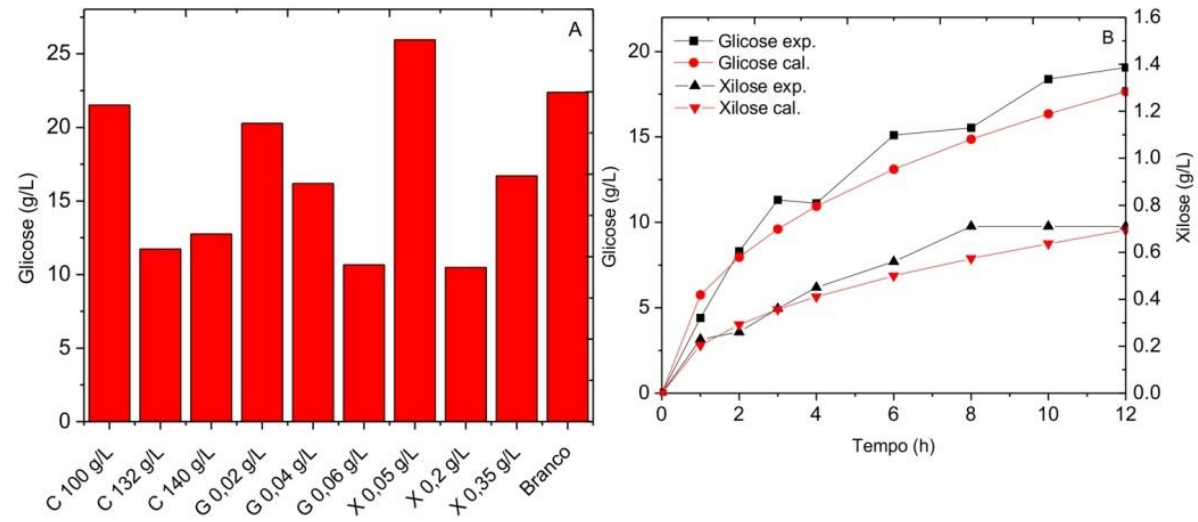

Figura 3. A - ensaios de inibição, B - modelo ajustado para 15 FPU/g celulose e $4 \%$ de celulose inicial.

De acordo com os resultados cinéticos onde se observa que a produção de glicose se mantem constante a partir de 75 FPU, os ensaios de adsorção foram dimensionados e foi comprovado que, com 75 FPU a enzima alcança o valor máximo de saturação. Desta forma, utilizando o software STATISTICA 7.0 foi possível encontrar os valores de Emax de 0,018 (g proteína/g celulose) e Kads de 23,28 (L/g proteína) por regressão não linear.

Para os ensaios de reatividade foi encontrada uma alta discrepância entre a reatividade ideal de 1,0 no tempo zero e a reatividade real no tempo de 15 min. Levando em consideração este resultado, a estrutura complexa da matriz lignocelulósica e a razão entre celulose (amorfa/cristalina) na molécula de celulose, a regressão $(\mathrm{C} / \mathrm{CoxRc})$ foi feita sem o tempo zero e a reatividade encontrada foi de 0,27 e, portanto, considerada no modelo aqui proposto.

Para os ensaios de inibição foi encontrado inibição no ensaio que leva em consideração a reação (celulose - glicose), para os outros ensaios não foi encontrado inibição nas condições 
estudadas. Observa-se que as constantes testadas na inibição da reação (celobiose - glicose) são bem maiores para glicose e xilose, caracterizando uma baixa inibição nesta rota. Desta forma, as inibições para glicose e xilose aqui determinadas foram consideras nas de reação $r_{1}$. Como é mais provável de que a celobiose iniba a reação (celulose - celobiose) do que (celobiose - glicose) a constante de inibição aqui encontrada foi considerada, também, na taxa de reação $r_{1}$.

Os valores de anergia de ativação foram de $9765 \mathrm{cal} / \mathrm{mol}$ na reação (celulose - glicose) em um ensaio feito por 3 hs e 2516 para a reação (celobiose - glicose) em um ensaio realizado por 10 min., pois em 3 horas todas os ensaios variando temperatura apresentaram conversão máximo de substrato a produto impedindo os cálculos da energia de ativação. Pelos resultados pode-se ter uma ideia que a maior energia de ativação é na etapa (celulose - celobiose) na reação global (celulose - glicose).

Tomando como base as publicações e os ensaios aqui realizados, um modelo matemático foi ajustado para a produção de glicose, a partir de bagaço de cana-de-açúcar submetido a um pré-tratamento físico químico, pelo processo de hidrólise enzimática. $\mathrm{O}$ modelo levou em consideração as taxas de reações $r_{1}, r_{2}, r_{3}$ e $r_{4}$.

Como a reação direta (celulose - glicose) é menos provável de acontecer, ou seja, pouca glicose é produzida de forma direta sem o intermediário celobiose mesmo que haja apenas as enzimas celulases e no caso deste estudo, existe uma alta atividade de celobiase, esta reação levou em consideração apenas a inibição de glicose e um pequeno valor da taxa constante de reação $\left(k_{2}\right)$. A taxa de reação $r_{2}$ levou em consideração as inibições por celobiose, glicose e xilose de acordo com os resultados dos ensaios experimentais de inibição como já comentado. A taxa de reação $r_{3}$ levou em consideração as inibições porcelobiose, glicose e xilose.Entretanto, a inibição de celobiose na taxa $r_{3}$ ficou expressa de forma diferente no modelo que é semelhante ao de Michaelis-Menten onde se tem um sistema homogêneo e a enzima não e adsorvida no substrato.Nas outras taxas, por se tratar de um sistema heterogêneo, foi preciso levar em consideração a adsorção da enzima ao substrato e sua reatividade. Para a taxa de reação $r_{4}$, foi levado em consideração apenas a inibição da xilose no processo, a razão (xilose/hemicelulose) foi de $(1 / 1)(\mathrm{m} / \mathrm{m})$ e a enzima que atuou no processo foi as celulases agindo na desestruturação de matriz lignocelulósica, por isso não foi levado em consideração a reatividade da hemicelulose. Entretanto, esta reação (hemicelulose - xilose) precisa ser melhor estudada. Por fim, o balanço de enzimas leva em consideração as celulases adsorvidas, celulases livres e $\beta$-glicosidasetotal que se encontra livre. $\mathrm{O}$ modelo ajustado está descrito nas Equações de 1 a 14.

$$
\text { Celulose } \frac{r_{1}}{G_{2}, G, X} \text { Celobiose } \frac{r_{3}}{G, X} \text { Gli } \cos e
$$

Celulose $\frac{r_{2}}{G}$ Gli $\cos e$

$$
\text { Hemicelulose } \frac{r_{4}}{X} \text { Xilose }
$$




$$
E T_{1}=E B_{1}+E F_{1}+E T_{2}
$$

$$
E B_{1}=\frac{E \max K a d s E F_{1} C}{1+\left(K a d s E F_{1}\right)}
$$

$$
r_{1}=\frac{k_{1} E B_{1} \text { KadsRcC }}{1+\left(\frac{G_{2}}{K_{1} I G_{2}}+\frac{G}{K_{1} I G}+\frac{X}{K_{1} I X}\right)}
$$

$$
r_{2}=\frac{k_{2} E B_{1} K a d s R c C}{1+\left(\frac{G}{K_{2} I G}\right)}
$$

$$
r_{3}=\frac{k_{3} E T_{2} G_{2}}{K_{3} M\left(1+\frac{G}{K_{3} I G}+\frac{X}{K_{3} I X}\right)+G_{2}}
$$

$$
r_{4}=\frac{k_{4} E B_{1} H}{1+\left(\frac{X}{K_{4} I X}\right)}
$$

$$
\frac{d C}{d t}=-r_{1}-r_{2}
$$

$$
\frac{d G_{2}}{d t}=1,056 r_{1}-r_{3}
$$

$$
\frac{d G}{d t}=1,111 r_{1}+1,053 r_{2}
$$

$$
\frac{d H}{d t}=-r_{4}
$$

$$
\frac{d X}{d t}=r_{4}
$$

De acordo com o teste do $X^{2}$ o valor tabelado foi de $(2,73)$ para $(\alpha=0,05$ e $d=8)$ e os valores para a condição da Figura 3B foi de 1,33 para a produção de glicose e 0,06 para a produção de xilose. Valos de $X^{2}$ calculados menores que o valortabelado indica um ajuste entre os dados experimentais e os calculados. $\mathrm{O}$ mesmo ajuste foi encontrado para as outras 
condições aqui testadas com exceção de alguns comportamentos da celobiose onde se encontrou uma maior dificuldade de se ajustar o modelo. Entretanto, como a celobiose é apenas um intermediário da reação (celulose - glicose) um ajuste perfeito desta curva não é tão necessário um vez que os ajustes para celulose, glicose, hemicelulose e xilose foram satisfatórios. Os valores das constantes cinéticas encontradas no modelo estão apresentados na Tabela 2.

Tabela 2. Valores das constantes do modelo de hidrólise enzimática.

\begin{tabular}{|c|c|c|c|}
\hline Parâmetrs & Valores & Unidades & Denominação \\
\hline \multicolumn{4}{|c|}{ Parâmetros constantes } \\
\hline Emax & 0,018 & $g / g$ & Máximo de enzimas celulases adsorvida na celulose \\
\hline Kads & 23,28 & $\mathrm{~L} / \mathrm{g}$ & Constante de adsorção das enzimas celulases na celulose \\
\hline $\mathrm{k}_{1}$ & 95,0 & L/g.h & Taxa constante de reação (celulose - glicose) \\
\hline $\mathrm{Rc}$ & 0,27 & & Reatividade do substrato (celulose) \\
\hline $\mathrm{K}_{1} \mathrm{IG}_{2}$ & 132,0 & $g / L$ & Constante de inibição da celobiose \\
\hline $\mathrm{K}_{1} \mathrm{IG}$ & 0,04 & $\mathrm{~g} / \mathrm{L}$ & Constante de inibição da glicose \\
\hline $\mathrm{K}_{1} \mathrm{IX}$ & 0,2 & $\mathrm{~g} / \mathrm{L}$ & Constante de inibição da xilose \\
\hline $\mathrm{k}_{2}$ & 15,0 & L/g.h & Taxa constante de reação (celulose - glicose) \\
\hline $\mathrm{K}_{2} \mathrm{IG}$ & 1,0 & $\mathrm{~g} / \mathrm{L}$ & Constante de inibição da celobiose \\
\hline $\mathrm{k}_{3}$ & 300,0 & $1 / \mathrm{h}$ & Taxa constante de reação (celobiose-glicose) \\
\hline $\mathrm{K}_{3} \mathrm{M}$ & 7,0 & $g / L$ & Constante de saturação da celobiose \\
\hline $\mathrm{K}_{3} \mathrm{IG}$ & 20,0 & $\mathrm{~g} / \mathrm{L}$ & Constante de inibição da glicose \\
\hline $\mathrm{K}_{3} \mathrm{IX}$ & 250,0 & $\mathrm{~g} / \mathrm{L}$ & Constante de inibição da xilose \\
\hline $\mathrm{k}_{4}$ & 11,0 & L/g.h & Taxa constante de reação (hemicelulose - xilose) \\
\hline $\mathrm{K}_{4} \mathrm{IX}$ & 0,009 & $\mathrm{~g} / \mathrm{L}$ & Constante de inibição da xilose \\
\hline \multicolumn{4}{|c|}{ Parâmetros variáveis } \\
\hline $\mathrm{C}$ & - & $g / L$ & Celulose \\
\hline $\mathrm{G}_{2}$ & - & $\mathrm{g} / \mathrm{L}$ & Celobiose \\
\hline $\mathrm{G}$ & - & $\mathrm{g} / \mathrm{L}$ & Glicose \\
\hline $\mathrm{H}$ & - & $\mathrm{g} / \mathrm{L}$ & Hemicelulose \\
\hline $\mathrm{X}$ & - & $\mathrm{g} / \mathrm{L}$ & Xilose \\
\hline ET & - & $\mathrm{g} / \mathrm{L}$ & Enzimas totais \\
\hline $\mathrm{EB}_{1}$ & - & $\mathrm{g} / \mathrm{L}$ & Enzimas celulases adsorvidas \\
\hline $\mathrm{EF}_{1}$ & - & $\mathrm{g} / \mathrm{L}$ & Enzimas celulases livres \\
\hline $\mathrm{ET}_{2}$ & - & $\mathrm{g} / \mathrm{L}$ & Enzima $\beta$-glicosidasetotal livre \\
\hline
\end{tabular}

\section{CONCLUSÃO}

Em acordo com o apresentado conclui-se que o modelo proposto para descrever a hidrólise enzimática do bagaço de cana-de-açúcar representa de forma fenomenológica,satisfatoriamente, o sistema aqui estudado. 


\section{REFERÊNCIAS}

BELKACEMI,K.; HAMOUDI, S.Enzymatic hydrolysis of dissolved corn stalkhemicelluloses: reaction kinetics and modeling.J ChemTechnolBiotechno,v. 78, p. 802808, 2003.

GALBE, M.; ZACCHI, G.A review of the production of ethanol from softwood.ApplMicrobiolBiotechnol, v. 59, p. 618-628, 2002.

GHOSE, T. K. Measurement of cellulose activities. Pure Appl Chem.v. 59, p. 257-268, 1987.

GOUVEIA, E. R.; NASCIMENTO, R. T.; SOUTO-MAIOR, A. M.; ROCHA, G. J. M. Validação de metodologia para a caracterização química de bagaço de cana-de-açúcar.Quim Nova, v.32, p. 1500-1503, 2009.

GUO, G. L.; HSU, D. C.; CHEN, W. H.; CHEN, W.H.; HWANG, W. S. Characterization of enzymatic saccharification for acid-pretreated lignocellulosicmaterials with different lignin composition. Enzyme MicrobTechnol, v. 45, p. 80-87, 2009.

KADAM, K. L.; RYDHOLM, E. C.; MCMILLAN, J. D. Development and Validation of a Kinetic Model for EnzymaticSaccharification of Lignocellulosic Biomass.BiotechnolProg,v. 20, p. 698-705, 2004.

KHODAVERDI, M.; JEIHANIPOUR, A.; KARIMI, K.; TAHERZADEH, M. J. Kinetic modeling of rapid enzymatic hydrolysis of crystallinecellulose after pretreatment by NMMO, J IndMicrobiolBiotechnol. v. 39, p. 429-438, 2012.

LIAO, W.; LIU, Y.; WEN, Z.; FREAR, C.; CHEN, S. Kinetic Modeling of Enzymatic Hydrolysis of Cellulose in Differently Pretreated Fibers From Dairy Manure. BiotechnolBioeng, v. 101, p. 441-451, 2008.

LYND, L.; WEIMER, P.; ZYL, W.; PRETORIUS, A. Microbial cellulose utilization: fundamentals and biotechnology. MicrobiolMol Bio Rev, v. 66, p. 506-577, 2002.

MORALES-RODRIGUEZ,R.; GERNAEY, K. V.; MEYER,A. S.; SIN, G.A Mathematical Model for Simultaneous Saccharification andCo-fermentation (SSCF) of C6 and C5 Sugars.Chinese Journal of Chemical Engineering, v. 19, p. 185-191, 2011.

TING, C. L.; MAKAROV, D. E.; WANG, Z. G.A Kinetic Model for the Enzymatic Action of Cellulase, J. Phys. Chem,v. 113, p. 4970-4977, 2009.

VASQUEZ, M. P.; SILVA, J. N. C.; SOUZA, M. B. Jr.; PEREIRA, N. Jr. Enzymatic hydrolysis optimization to ethanol production by simultaneous saccharification and fermentation. ApplBiochemBiotechnol, v. 136-140, p. 141-154, 2007.

ZHOU, J.; WANG, Y. H.; CHU, J.; LUO, L. Z.; ZHUANG, Y. P.; ZHANG, S. L. Optimization of cellulasemixture for efficient hydrolysis of steam-exploded corn stover by statistically designed experiments. BioresourTechnol, v. 100, p. 819-825, 2009. 